\title{
Partition Based Cascaded Generator Scheduling with Constraints for Large Power Networks
}

\author{
Zakia Asad, Mohammad Asad Rehman Chaudhry, and Deepa Kundur \\ z.asad@mail.utoronto.ca, asadchau@ie.ibm.com,dkundur@comm.utoronto.ca
}

\begin{abstract}
Power systems are on the cusp of a rapid technological, economic and environmental evolution. Classical problems must naturally adapt to new requirements taking into account enormous growth in the number of distributed generation plants. Physical and computational constraints within the new landscape must be revisited. In this paper, we focus on the generator scheduling problem in smart grid incorporating the novel constraints of transmission line capacity limits and policy to provide a more comprehensive view for planning in the smart grid. Given the mammoth size of the power networks, we propose a computationally efficient partition-based scheduling scheme. A case study based on IEEE 300 bus test system shows the advantage of the proposed scheme.
\end{abstract}

\section{INTRODUCTION}

The paradigm shift for the power systems from relying on few large central power plants (mainly coal, hydal, and nuclear) towards a large number of small distributed-generation power plants has increased the complexity of fundamental problems like generator scheduling to many folds. In many such scenarios, the solutions that are tractable for traditional setups become "practically" intractable [1]. Therefore there is a need for developing computationally efficient solutions that are "practically" tractable for very large problem instances. We propose to use a divide-and-conquer type approach which partitions a power network into smaller and efficiently solvable sub-networks. Such partitioning strategies have been shown to be much powerful when dealing with systems of mammoth sizes and have applications in a large array of systems (for few examples see [2], [3], [4]). However, partitioning a power network into any arbitrary set of sub-networks might not be useful, rather partitions based on the problem specification are required since we might loose the solutions quality otherwise.

In this paper we focus on generator scheduling under a set of emerging constraints imposed by deregulation in power market. Scheduling generation plants to meet load demands at least operating cost has historically been a cardinal problem in power systems. A number of tools including prioritylists, dynamic programming, integer programming, branch and bound, simulate annealing, Lagrangian relaxation, and genetic algorithms (see [5],[6] and references therein) have been applied to solve this known NP-hard problem [7]. In its classical setting, this unit commitment problem involves selection of generation units to supply the forecasted load in

Zakia Asad, and Deepa Kundur are associated with The Edward S. Rogers Sr. Department of Electrical and Computer Engineering, University of Toronto. Mohammad Asad Rehman Chaudhry is associated with IBM Research and Hamilton Institute. The authors would like to acknowledge the support of Schlumberger Foundation, and the Irish Research Council. a way that minimizes cost over a required planning horizon while leaving a specified margin of spinning reserve.

Traditionally, the unit commitment problem has accounted for some system constraints like individual startup delays, generation costs, and physical operational constraints. Shift to a "smarter" grid in a deregulated market results in a fluctuating technical, political and financial landscape in which decisions are constrained by additional issues including policy and transmission line bottlenecks. For instance, although it may take one year to build a wind farm, it can take five years to build the necessary transmission lines needed to carry its power to cities [8]. In addition, the smart grid vision incorporates consumer-driven and regulator-driven policies such as priority of renewable sources resulting in novel system constraints. Furthermore, the burgeoning cyber infrastructure promotes high performance computing applications for a more optimized grid necessitating that distributed computing and complexity be considered. Moreover, move towards distributed generation with many small mass-produced energy sources has proportionally resulted in hugely increased complexity of the generator scheduling problem. Hence, there is need for developing the solutions that can scale-well with the humongous increase in the number of generation points. We assert that an important step in smart grid development involves revisiting and reformulating classical problems to suit emerging constraints.

In this vein, we consider the generator scheduling problem in which we integrate new requirements. Specifically, we address: 1) transmission line constraints involving line overloads that arise from usage by multiple generating points, 2) policy constraints which deal with prioritization of generation source classes for specific load areas, and 3) delay constraints including startup lags. We call this the constrained generation schedule (CGS) problem. To address the needs of smart grid systems, we associate a time-varying cost of operation to each generating source, for example, to reflect the changing cost of solar or wind power. An instance of the CGS problem consists of a number of generating points, a set of transmission lines, and a set of load areas. Each generating point can represent a collection of generators or virtual power plants (VPPs) each with an associated delay. Each load area consists of a set of geographical regions with policy-driven preferences regarding the choice of generating points and has a forecasted demand. There is a price tag offered by generation companies (GenCos) for each generating point to serve the forecasted demand of a specific load area. Transmission lines that are either owned by transmission companies (TxCos) or have 
transmission rights reserved for specific GenCos or consumer areas are a shared resource with limited capacity. We transform the system of aforementioned constraints into an equivalent graph representation and partition the power network to reduce the complexity of the CGS problem, where each partition comes up with its own scheduling in a cascaded fashion. Example I-B elaborates it further.

\section{A. Motivation and Contributions}

Although intelligent generation scheduling (involving unit commitment [9], [10] and economic dispatch [11]) has been widely studied, but classically generator scheduling has not accounted for policy and transmission line capacity constraints often leaving its inclusion to the next stage of economic dispatch. However, the growing trend in power systems is one in which these limitations must be accounted for early on during the scheduling phase to provide a more comprehensive view [12]. Therefore, research has also been done for the generator scheduling in the presence of the security constraints [13] and transmission line capacity constraints [14], but the drift towards "smarter" grid calls for inclusion of policy based constraints which is addressed in this paper. Moreover, to address the tremendous growth in the number of generating points research has also been done to solve the generator scheduling problem for real-life large-scale power systems (e.g., see [1]) that are mostly heuristic solutions. However, question of developing a more concrete deterministic solution for large power network is still open.

Scheduling generation to match demand while simultaneously meeting constraints involves extensive combinatorics due to the discrete nature of the problem; a generator is either selected or not. In general, choosing the least cost combination of generating points requires exhaustive search. An alternative approach could relax the integer constraints imposed by the problem and solve it via linear programming; however, such a solution may lack optimality and in some cases rounding might result in an infeasible solution [15]. Thus, in this work we propose an approach for combinatorial optimization that aims to leverage the natural symmetry and structure of the CGS problem that is suitable for distributed computing while providing insight on issues of computational complexity. We focus on the application of matriod theory [16] to the smart grid scheduling problem. The structure of matroids enables one to effectively rule out large groups of generator sets for scheduling with a simple test instead of sifting through all possible combinations.

We propose a novel computationally efficient partitionbased scheme for generator scheduling in large scale power networks. In the proposed scheme generators are partitioned based on their constraint-driven correlation with each other. We then use an cascaded scheduling algorithm to schedule the generators in closely correlated partitions, and the generators in rest of the partitions are scheduled independently. Moreover, a case study based on IEEE 300 bus power system [17] shows the advantage of our proposed scheme.

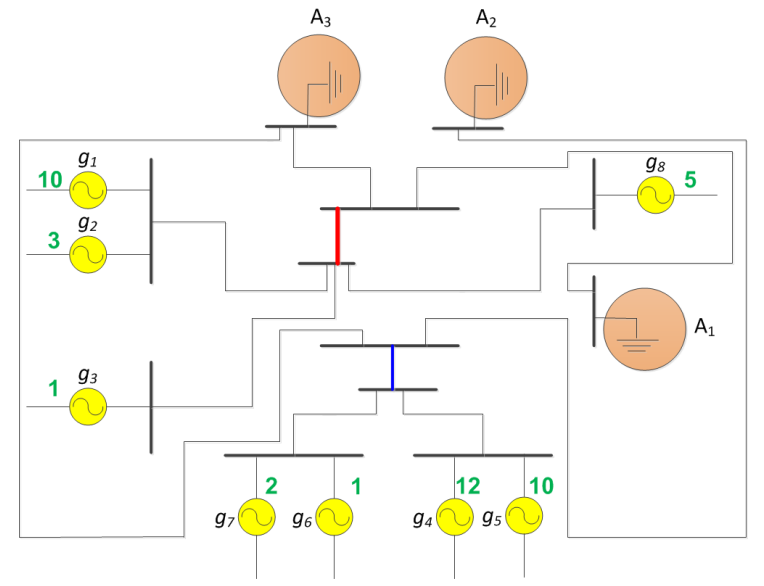

Fig. 1. A 12 bus power system with eight generating points $g_{1}, \cdots, g_{8}$ and there load areas to be served $A_{1}, A_{2}, A_{3}$.

\section{B. Example Case Study: 12-Bus Power System}

Consider a 12-bus power system, that we revisit throughout this paper, with generating points $g_{1}, \cdots, g_{8}$, load areas to be served $A_{1}, A_{2}, A_{3}$, and a set of two bottleneck transmission lines $\gamma_{1}$ (in red), $\gamma_{2}$ (in blue) shown in Fig. 1. The sum from any group of two or more generating point outputs exceeds the transmission line capacities of $\gamma_{1}$ and $\gamma_{2}$, and therefore prohibits simultaneous use by more than one generating point. The cost of the each generating point is shown in green. A careful study reveals the following facts.

If selected for dispatch, generating points $G_{\gamma_{1}}=$ $\left\{g_{1}, g_{2}, g_{3}, g_{8}\right\}$ need to use one common transmission line $\gamma_{1}$ and thus cannot be simultaneously scheduled. Similarly, $G_{\gamma_{2}}=\left\{g_{4}, g_{5}, g_{6}, g_{7}\right\}$ cannot be simultaneously scheduled for dispatch on $\gamma_{2}$. Thus the transmission line constraints impose that no two generating points from $G_{\gamma_{1}}$ and $G_{\gamma_{2}}$ be scheduled to use $\gamma_{1}$ and $\gamma_{2}$, respectively, at the same time. Moreover, due to policy matters each load area has preference of generating points. In this example, $A_{1}$ chooses $G_{A_{1}}=\left\{g_{1}, g_{2}, g_{3}\right\}, A_{2}$ opts for $G_{A_{2}}=\left\{g_{4}, g_{5}\right\}$, and area $A_{3}$ must fulfill its demand with $G_{A_{3}}=\left\{g_{6}, g_{7}, g_{8}\right\}$. Thus, within the scope of the planning horizon, the transmission line and policy constraints dictate selection of only one generating point from each of $G_{A_{1}}, G_{A_{2}}$, and $G_{A_{3}}$ for load areas $A_{1}, A_{2}$ and $A_{3}$ respectively. In this example, we assume that any generating point within $G_{A_{i}}$ can accommodate the forecasted load for $A_{i}$; if that is not the case then more than one generating point from the set can be selected.

\section{MODEL}

We consider scheduling from a set of $n$ generating points $G=\left\{g_{1}, \cdots, g_{n}\right\}$ over $\ell$ transmission lines $\Gamma=\left\{\gamma_{1}, \cdots, \gamma_{\ell}\right\}$ for $k$ load areas $A_{1}, \cdots, A_{k}$ and a planning horizon $T$. $W_{i}, L_{j}$ and $D_{m}$ denote the output of $g_{i}$, capacity limit of transmission line $\gamma_{j}$ and the forecasted load demand for area $A_{m}$, respectively.

Each generating point is associated with a time-dependent generation cost $c: G \times\{0,1, \cdots, T-1\} \longrightarrow \mathbb{R} \geq 0$ 


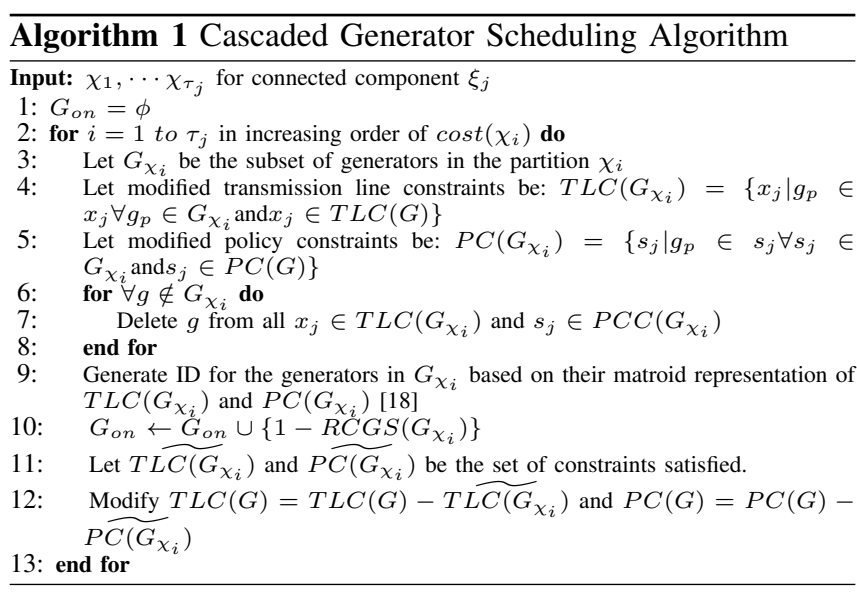

that assigns non-negative costs to each generating point $g_{i}$ over all time units $t_{j} \in\{0,1, \cdots, T-1\}$ such that $c\left(g_{i}, t_{j}\right)$ refers to the generation cost of $g_{i}$ at $t_{j}$. In addition, each generating point $g_{i}$ is associated with a generation delay $d\left(g_{i}\right) \in\{0,1, \cdots\}$ to reflect wait times in startup. If $g_{i}$ is allocated a desired transmission line at $t_{j}$, it implies that the actual time when $g_{i}$ couples to the transmission line is $d\left(g_{i}\right)+t_{j}$.

An allocation at time $t_{i}$ denoted $a_{t_{i}}$ is a set of generating points that are allocated their desired transmission line at time $t_{i}$. A schedule is defined to be the set of allocations at times $0,1, \cdots, T-1$ for a planning horizon of $T$. A feasible schedule is a schedule that satisfies the following three constraints:

1) Transmission line constraints: are described by a family of subsets of $G, T L C(G)=\left\{x_{1}, \cdots, x_{R} \mid x_{i} \subseteq\right.$ $G \forall i\}$ where set $x_{i}$ contains all generating points that require line $\gamma_{i}$ but have conflicts such that their total output exceeds the line capacity $L_{i}$. Thus, in a feasible schedule if $g_{i}, g_{j} \in x_{v}$ and $g_{i}$ is allocated $\gamma_{v}$ at time $t_{m}$, and $g_{j}$ is allocated $\gamma_{v}$ at $t_{l}$, then $d\left(g_{i}\right)+t_{m} \neq d\left(g_{j}\right)+t_{l}$.

2) Delay constraints: In a feasible allocation and for a planning horizon $T$, a generating point $g_{i}$ can only be allocated its required transmission line at time $t_{j}$ if $d\left(g_{i}\right)+t_{j} \leq T-1$.

3) Policy constraints: are described by a family of subsets of $G$ denoted $P C(G)=\left\{s_{1}, \cdots, s_{k}\right\}$ consisting of distinct (non-overlapping) policy sets $s_{i} \subseteq G$ defined for each load area $A_{i}$ and representing the set of generating points opted for meeting its demand. We say $A_{i}$ has been provided the service if at least one generating point in $s_{i}$ has been allocated its desired transmission line. For each $s_{i}$ we define a variable $f\left(s_{i}\right) \in\{0,1\}$, where $f\left(s_{i}\right)=1$ if area $A_{i}$ has been provided the service, and $f\left(s_{i}\right)=0$ otherwise. Since a goal is to provide service to as many service areas as possible, formally we require maximization of $\sum_{s_{i} \in P C(G)} f\left(s_{i}\right)$.

The cost of an allocation $a_{t_{i}}$ is defined to be the sum of the generation costs of all the generating points in $a_{t_{i}}$ given by: $\operatorname{cost}\left(a_{t_{i}}\right)=\sum_{g_{j}: g_{j} \in a_{t_{i}}} c\left(g_{j}, t_{i}\right)$. Subsequently, the cost of a schedule $Q$ is given by of the sum of costs over all its allocations given by: $\operatorname{cost}(Q)=\sum_{a_{t_{i}}: a_{t_{i}} \in Q} \operatorname{cost}\left(a_{t_{i}}\right)$.

Definition 1: Constrained Generation Scheduling (CGS)

Problem Find a least cost feasible schedule; that is, no other feasible schedule has a lower cost.

Informally, we can say that the objective of the CGS problem is to provide service to the maximum possible number of service areas while respecting their policy initiatives without violating the transmission line and delay constraints such that the solution has the least cost amongst all constrained possibilities.

For easy excursion through the paper we focus on the 1 $R C G S$ problem, as defined below, for the rest of the paper. The solution can then be extended to the general problem using extended sets [18]. In the restricted constrained generation scheduling (RCGS) problem we assume that each transmission line has equal capacity (i.e., $L_{i}=L_{j}, \forall i, j$ ) and each generating point has same level of output (i.e., $\left.W_{i}=W_{j}, \forall i, j\right)$. Furthermore, we assume that the capacity of the transmission lines is less than twice the output capacity of any one generating point. We start by presenting the solution to the 1-RCGS problem which exhibits the following additional restrictions: $T=1$, which naturally implies $d\left(g_{i}\right)=0 \forall i$ and $c\left(g_{i}, t_{j}\right)=c\left(g_{i}\right) \forall i, j$.

It is obvious that the the least cost schedule for the 1-RCGS problem, is same as least cost allocation at time $t_{i}=0$ since the planning horizon is just one time unit.

\section{Partition BASEd CASCADED Generator SCHEDULING}

As described in Section II, the transmission-line constraints and policy constraints can be presented in form of sets of subsets of generators. Our focus is to partition the generating points in a way such that the intersection of the constraints sets for any two partitions has least cardinality. To capture the constraint sharing between any two generating points we construct the Constrained Graph $C(V, E)$, a vertex-weighted undirected graph with vertex set $V$, and edge set $E$. Each vertex $v_{i} \in V$ is assigned a weight $w\left(v_{i}\right)$. Given an instance of 1-RCGS problem we define the Constrained Graph $C(V, E)$ as: $V:=G$ and $E:=\left\{\left(g_{i}, g_{j}\right) \mid g_{i}, g_{j} \in x_{p}, p=1, \cdots, R\right\} \cup$ $\left\{\left(g_{i}, g_{j}\right) \mid g_{i}, g_{j} \in s_{q}, q=1, \cdots, k\right\}$, and $w\left(v_{i}\right)=c\left(g_{i}\right)$. An example of the Constrained Graph for the 12-Bus example is shown in Fig. 2. Next we find connected components in the Constrained Graph. After that for each connected component we find the partitions using Sparsest Cut Clustering [19]. As a result of Sparsest Cut Clustering for each connected component of $C(V, E)$, say $\xi_{j}, \tau_{j}$ partitions $\chi_{1}, \cdots \chi_{\tau_{j}}$ are formed. Each partition consists of a number of generating points (vertices of $C(V, E)$ ). Define the cost of a partition $\chi_{i}$ as: $\operatorname{cost}\left(\chi_{i}\right)=\sum_{v_{j} \in \chi_{i}} w\left(v_{j}\right)$.

Without loss of generality, order the partitions such that $\operatorname{cost}\left(\chi_{1}\right) \leq \operatorname{cost}\left(\chi_{2}\right) \cdots \leq \operatorname{cost}\left(\chi_{\tau_{j}}\right)$. We call two partitions to be adjacent if they share one or more edges. For each connected component the proposed Constrained Generator Scheduling algorithm works in a cascaded fashion solving scheduling problem for one partition at a time in increasing order of the partition costs. The primary motive behind 


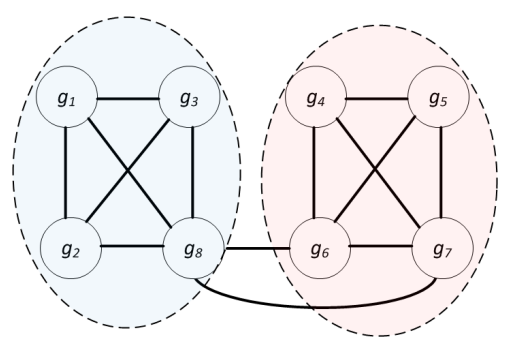

Fig. 2. Graph representation of the constraints imposed by the 12 bus power system.

cascaded scheduling for each connected component is that in certain scenarios different partitions might share a number of edges (representing one or both constraints) where solving the constrained generation scheduling for one partition solves a number of constraints that are needed to be removed for the adjacent partitions for avoiding repeatedly solving the same constraint in adjacent partitions. Furthermore, the choice of solving constrained generation scheduling in order of the costs of partition results in greedy optimization over the constraints shared among different partitions since solving the lesser cost partitions first might result in inclusion of lesser cost generator in the final schedule compared to its costly replacement in adjacent partition. Specifically Algorithm 1 Cascaded Scheduling Algorithm starts by initiating the set of generators selected for final schedule $G_{o n}$ to be $\phi$ (step 1) and then proceed with finding the locally optimal schedule for the partition of generators $G_{\chi_{i}}$ in increasing order of their costs. Steps 4-8 define the transmission line constraint set $T L C\left(G_{\chi_{i}}\right)$ and policy constraint set $P C\left(G_{\chi_{i}}\right)$ for partition $\chi_{i}$ by deleting the constraints over the generators not in the partition $\chi_{i}$ from $T L C(G)$ and $P C(G)$ respectively. Step 9 generate the IDs for the generators and Step 10 runs Algorithm $1-R C G S$ [18] for finding the allocation of generators and append it to $G_{o n}$. Step 12 modifies $T L C(G)$ and $P C(G)$ by deleting the constraints satisfied for the partition $\chi_{i}$ i.e., $T \widehat{L C\left(G_{\chi_{i}}\right)}$ and $\widehat{P C\left(G_{\chi_{i}}\right)}$. The same steps are repeated for rest of the partitions.

For the 12-bus example I-B, the sparsest cut partitions are $\chi_{1}=\left\{g_{1}, g_{2}, g_{3}, g_{8}\right\}$ and $\chi_{2}=\left\{g_{4}, g_{5}, g_{6}, g_{7}\right\}$ with the corresponding costs 19 and 25 respectively. Note that there is just one connected component for the Constrained Graph shown in Fig. 2.

Lemma 2: For each partition the schedule found in steps 3-10 of the Algorithm 1 is locally optimal.

Theorem 3: If for each connected component $\xi_{j},\left|\tau_{j}\right|=1$ then the Algorithm 1 provides the optimal solution.

Brief Sketch of Proof When the partitions are non-adjacent then the locally optimal schedule found for each partitions is globally optimal as well.

\section{Test System Study}

To evaluate the performance of the propose scheme on practical power systems we performed a simulation study using IEEE 300 bus test system [17]. IEEE 300 bus test system was developed by IEEE Test Systems Task Force in 1993, so

\begin{tabular}{|c|c|c|c|c|}
\hline No. of Partitions & 5 & 10 & 15 & 20 \\
\hline$\mu$ & 1.1390 & 1.6118 & 2.0282 & 2.4474 \\
\hline
\end{tabular}

TABLE I

it does not incorporate the smart grid scenario with a large number of generating points. To incorporate the smart grid scenario with a large number of generating points, we extended the number of generating points in this system which is a know practice (see for example [20]). To incorporate both the sub-optimality, and running time of the proposed scheme we used the cost-time product as the performance metric. More specifically, for each realization we calculated the product of the total cost of scheduling and the total running time. We compared the cost-time product of the proposed scheme with standard optimal solution. We define $\mu$ to be the ratio of the cost-time product of the proposed scheme and standard optimal solution. Table I shows average $\mu$ (average was taken over 100 random instances) versus number of partitions. $\mu>1$ imply better performance of the proposed scheme compared to standard optimal solution.

\section{REFERENCES}

[1] J. Álvarez, L. Meza, I. Guillén, and R. Gómez, "A heuristic algorithm to solve the unit commitment problem for real-life large-scale power systems," International Journal of Electrical Power \& Energy Systems, vol. 49, pp. 287-295, 2013.

[2] J. Pujol, V. Erramilli, and P. Rodriguez, "Divide and conquer: Partitioning online social networks," arXiv:0905.4918, 2009.

[3] I. Lendak, A. Erdeljan, and I. Rudas, "Algorithm for adaptive smart grid visualization," in IEEE ICIT 2013.

[4] I. Hänninen, W. Buckhanan, M. Niemier, and G. Bernstein, "Network on metachip architectures," in ACM NoCarC, 2012, pp. 5-10.

[5] S. Orero and M. Irving, "A genetic algorithm for generator scheduling in power systems," International Journal of Electrical Power \& Energy Systems, vol. 18, no. 1, pp. 19-26, 1996.

[6] X. Guan, P. Luh, and B. Prasannan, "Power system scheduling with fuzzy reserve requirements," IEEE Transactions on Power Systems, vol. 11, no. 2, pp. 864-869, 1996.

[7] C. L. Tseng, On Power System Generation Unit Commitment Problems, Dissertation, University of California at Berkeley, 1996.

[8] ," http://www.seco.cpa.state.tx.us/.

[9] A. Bhardwaj, N. Tung, and V. Kamboj, "Unit commitment in power system: A review," International Journal of Electrical and Power Engineering, vol. 6, no. 1, pp. 51-57, 2012.

[10] B.H. Chowdhury and S. Rahman, "A review of recent advances in economic dispatch," Power Systems, IEEE Transactions on, vol. 5, no. 4, pp. 1248-1259, 1990.

[11] H. Happ, "Optimal power dispatch: A comprehensive survey," IEEE Transactions on Power Apparatus and Systems, vol. 96, no. 3, 1977.

[12] D. Kirshen and G. Strbac, Fundamentals of power system economics, John Wiley \& Sons Ltd, 2004.

[13] K. Ng and J. Zhong, "Security-constrained dispatch with controllable loads for integrating stochastic wind energy," in IEEE PES ISGT, 2012.

[14] K. Zare and S. Hashemi, "A solution to transmission-constrained unit commitment using hunting search algorithm," in IEEE EEEIC.

[15] ," http://mat.gsia.cmu.edu/orclass/integer/node11.html.

[16] J.G. Oxley, Matroid theory, Oxford University Press, USA, 2006.

[17] ," http://www.ee.washington.edu/research/pstca/index.html/.

[18] Z. Asad, M. Chaudhry, and D. Kundur, "On the use of matroid theory for distributed cyber-physical-constrained generator scheduling in smart grid," Technical Report The Edward S. Rogers Sr. Department of Electrical and Computer Engineering, University of Toronto, 2013.

[19] D. Spielman and S. Teng, "A local clustering algorithm for massive graphs and its application to nearly linear time graph partitioning," SIAM Journal on Computing, vol. 42, no. 1, 2013.

[20] A. Diniz, "Test cases for unit commitment and hydrothermal scheduling problems," in IEEE PES GM 2010. 